\title{
A STUDY OF THE CARRIER CONDITION ASSOCIATED WITH TYPE II PNEUMONIA IN A CAMP OF THE CIVILIAN CONSERVATION CORPS ${ }^{1}$
}

\author{
By A. H. HARRIS AND H. S. INGRAHAM \\ (From the Division of Laboratories and Research, and the Division of Communicable \\ Diseases, New York State Department of Health, Albany)
}

(Received for publication August 21, 1936)

A veterans' camp of the Civilian Conservation Corps was opened in Madison County in New York State early in 1935; reforestation was the principal activity. The enrolled men were drawn in approximately equal numbers from civil life, from a veterans' camp in Vermont, and from one in New Jersey. A few came from a soldiers' home in a nearby county and a small number was transferred from other C.C.C. camps. Most of the men were between the ages of 37 and 49; the youngest was 32 and all but eight were under 50. The population had been in a continual state of flux. A large contingent from the camp in New Jersey arrived on November 21. From this date, however, until the investigation was concluded, on December 17, only one or two men entered.

During the latter part of October and early November, the men had been confined to barracks and to the recreation building, where they were in close contact throughout several rainy days. At this time, over half of them developed respiratory infections, which in many instances were persisting when the first investigation was undertaken on December 8.

The first case of pneumonia developed on November 9 , in the course of this epidemic of colds, in a camper who had been transferred from the New Jersey camp eight days previously. He was treated in the camp infirmary, the sputum was not typed, and following an uneventful recovery, he was discharged from camp. It is noteworthy that no Type II pneumonia had previously occurred at this New Jersey camp. Between November 12 and December 1, six other cases of pneumonia developed in the Madison County camp, and still another occurred in a nearby vil-

\footnotetext{
1 Presented at the meeting of the New York State Association of Public Health Laboratories, Poughkeepsie, New York, May 25, 1936.
}

lage; the individual in this instance was a bartender in a café which was a favorite rendezvous of the campers. All except the first patient were treated at the Cortland County Hospital where laboratory studies demonstrated the presence of Type II pneumococci. All of the patients recovered except one individual who, before the onset of his illness, suffered extreme exposure while under the influence of alcohol. Among those who recovered, Type II pneumococci were isolated once from the blood of one individual and on two separate occasions from that of another. The testing of a strain obtained from the latter patient, which was maintained in semisolid medium, revealed that it was highly virulent for mice; that is, mice weighing between sixteen and twenty-two grams, inoculated in duplicate with $0.00000001 \mathrm{cc}$. of an 18-hour broth culture died within forty-eight hours. None of the patients received antipneumococcus serum.

On December 8, 1935, an investigation was undertaken to determine the incidence and distribution of carriers of Type II pneumococci within the camp. Throats of the campers were cultured, and pertinent data were secured through the taking of individual histories.

Cultures were obtained at the rate of from fifteen to thirty a day, usually just before mess when the men were congregated in their respective barracks; care was taken in swabbing out the tonsillar crypts and in reaching as high in the nasopharynx as a straight swab would allow. The swabs were then placed immediately in Avery's broth, and were incubated for from four to seven hours at $37.5^{\circ} \mathrm{C}$.; of the resulting growth, from 0.3 to $0.5 \mathrm{cc}$., depending on the density, were inoculated into mice. The peritoneal exudates of those mice which died spontaneously were examined directly for the presence of Type II pneumococci by the Neufeld technic; the heart's blood, in each instance, was streaked on blood-agar plates, and after twenty-four hours' incubation, the resulting growth was examined directly by the 
Neufeld method.2 Most of the strains of pneumococcus Type II were then transferred to blood-agar slants, for confirmatory tests by the macroscopic tube agglutination method. Mice which failed to develop symptoms of illness within forty-eight hours were not examined. During the final survey in April, however, such animals were chloroformed and autopsied; in rare instances, pneumococci were isolated in this manner, but the types were of minor interest.

Among 142 individuals examined in the five barracks, twenty-seven were found to be carriers, an incidence of 19 per cent. Table I indicates

TABLE I

Distribution of cases of Type II pneumonia and carriers of Type II pneumococci

\begin{tabular}{c|c|c|c}
\hline \hline Barrack & $\begin{array}{c}\text { Number of } \\
\text { men in } \\
\text { barrack at } \\
\text { time of } \\
\text { survey * }\end{array}$ & $\begin{array}{c}\text { Number } \\
\text { of cases }\end{array}$ & $\begin{array}{c}\text { Number } \\
\text { of carriers }\end{array}$ \\
\hline II & 34 & 2 & 5 \\
III & 31 & 2 & 4 \\
IV & 30 & 1 & 6 \\
V & 28 & 1 & 4 \\
\hline
\end{tabular}

* All but seven men were studied.

the relatively even distribution among the five barracks of the cases of Type II pneumonia and of individuals carrying pneumococci of that type in the throat.

Among seven officers examined, who were living in the officers' quarters, two whose special duties brought them into intimate contact with the enrolled men were found to be carriers. No carriers were discovered among nine foresters living in the forestry quarters or among six men on special duty scattered in various other buildings. Only one of the three men in the infirmary convalescing from Type II pneumonia proved to be a carrier. Two healthy bartenders at the café mentioned previously were not found to be carriers. The relationships between the carrier state and upper respiratory infection as well as between the carrier state and history of contact were not striking. Of the carriers, 30 per cent were suffering from some form of upper respiratory infection at the time of the investigation, 40 per cent gave a history of a recent cold, and 30 per

\footnotetext{
2 The pneumococcus typing and virulence tests during these surveys were undertaken by Miss Florence $M$. Varley.
}

cent gave no such history; 25 per cent of the carriers recalled intimate contact with one or more of the patients having pneumonia immediately prior to or during the illness. Of the noncarriers, 21 per cent were suffering from upper respiratory infection, 31 per cent gave a history of a recent cold, and 48 per cent gave no such history; 16 per cent of the noncarriers recalled contact with one or more of the pneumonia patients. It was possible only in rare instances to formulate a definite idea as to the source of the Type II pneumococci in a given case or carrier, since the opportunity for chance contact in the mess hall and general assembly room was great.

An incident of considerable interest was the subsequent development of a case of Type II pneumonia in barrack Number IV, on December 21 , three weeks after the onset of the next preceding case, and only six days after the man's throat had been cultured. He had not been found to be a carrier of Type II pneumococci, but of Type XXI ; however, his bed was next to that of a Type II carrier.

In order to secure information relative to the persistence of the carrier condition, a second survey was undertaken. The camp in Madison County was disbanded early in 1936, and most of the men were transferred to other companies in two camps located in Orange County, where they were engaged in flood control. The nature of their work entailed considerable exposure, which might be expected to favor the development of respiratory infections. However, no such epidemic had occurred since the beginning of the year. On February 26, twenty of the original carriers and three of the men who had recovered from Type II pneumonia were again examined to determine whether or not a sufficiently large number of them were still harboring the Type II pneumococci to warrant making a general survey. From the throats of nine of the original carriers and two of the recovered cases, Type II pneumococci were isolated. It was learned at this time that although a few sporadic cases of pneumonia had occurred in these camps, none of them had been incited by Type II pneumococci. In view of the possibility that these strains of Type II pneumococci might be of comparatively low virulence, tests were performed with the eleven strains isolated at this time from the heart's blood of each 
mouse which had been inoculated with Avery's broth cultures, and stored at $8^{\circ} \mathrm{C}$. for twelve days in serum semisolid medium (1). All of the strains were found to be highly virulent; mice inoculated in duplicate with $0.00000001 \mathrm{cc}$. of a 14-hour broth culture, died within forty-eight hours; pour plates of this dilution indicated that an average of five microörganisms had been injected.

In conjunction with these studies of the virulence of the eleven strains of Type II pneumococci, the factor of individual host resistance was investigated. $^{3}$ With the blood serum which had been obtained from the three recovered cases and from all but one of the twenty original ${ }^{4}$ carriers who were studied at this time, mouse protection tests were performed against pneumococcus Type II. With sera of selected individuals in this group, similar tests were made using pneumococcus Type I. Twenty of these sera were also tested for agglutination and precipitation. For purposes of control, the sera of ten normal men, who gave no history of pneumonia, who were of approximately the same age as the campers, but who had no connection with the camps, were similarly tested. Throat cultures obtained from this group of control individuals, at the time the blood was collected, failed to reveal the presence of pneumococci Types I or II.

In the mouse protection tests, $0.1 \mathrm{cc}$. of serum was tested with different doses of a 16-hour culture of the standard strains, Type II (Number 53) and Type I (Number 1). The dilutions of culture and of serum were prepared so that the required amount of each was contained in $0.5 \mathrm{cc}$. The $0.5 \mathrm{cc}$. of diluted culture and the $0.5 \mathrm{cc}$. of diluted serum were thoroughly mixed in the barrel of the syringe and immediately injected into the peritoneum of a mouse weighing from eighteen to twenty-two grams. Two dilutions of culture were used in each test, and three mice for each dilution. Those

3 These serological tests were performed at the central laboratory in Albany under the supervision of Miss Jessie L. Hendry.

4 "Original carriers" refers to those men who were shown to be carriers of Type II pneumococci during the December survey.

"Persistent carriers" refers to those original carriers who were again shown to be carriers of Type II pneumococci in February.

"Former carriers" refers to those original carriers who, by February, had apparently ceased to be carriers of Type II pneumococci. mice surviving for ninety-six hours or longer were considered protected by the serum. Heart's blood cultures were made at autopsy. Determinations of the minimal fatal dose (M.F.D.) made with each test according to the method described in a recent paper by Kirkbride, Hendry, and Murdick (2) indicated that the injection of one microörganism was sufficient to kill. In most instances, the results presented were obtained in two tests; in the others, three or only one was necessary.

While all of the twenty-two sera were tested against the Type II standard culture, only thirteen were tested with the Type I culture; these thirteen included sera from five men who had been inoculated in 1934 with Type I and Type II soluble antigen (an active form of the polysaccharide of Heidelberger and Avery) as a prophylactic measure against pneumonia of these types (3) ; of the five, three were persistent carriers and two were former carriers. The ten control sera were tested against both the Type I and Type II strains.

The results of these protection tests are summarized in Table II. The serum from only one of the three cases which had recovered from Type II pneumonia protected against as much as 100 M.F.D. of the Type II culture. On the other hand, sera from seven of the eleven former carriers and from five of the eight persistent carriers protected against 100 or more M.F.D. of the Type II culture; among the sera showing a significant degree of protective activity, those from two former and two persistent carriers, none of whom had received the immunizing dose, protected against as much as 10,000 M.F.D.; indeed, the sera from only three of the five who had received the Felton antigens protected against as much as 100 M.F.D. Only two of the ten control sera protected against as much as 100 M.F.D. In corresponding tests with the Type I culture, the serum from not one of the three recovered cases and from only one of the five carriers, none of whom had received the Felton antigens, afforded protection against as much as 100 M.F.D. In contrast, the sera from three of the five carriers who had received the Felton antigens protected against 100 or more M.F.D., and of these three, one protected against 10,000 M.F.D. Only two of the ten control sera protected against as much as 100 M.F.D.

All but two of the sera were of sufficient amount to permit agglutination tests in which cell 
TABLE II

Results of potency tests of sera obtained February 26, 1936, from original carriers

\begin{tabular}{|c|c|c|c|c|c|c|c|c|c|c|c|c|c|c|c|}
\hline \multirow[t]{2}{*}{ Condition } & \multirow{2}{*}{\multicolumn{2}{|c|}{$\begin{array}{c}\text { Number } \\
\text { tested }\end{array}$}} & \multicolumn{6}{|c|}{$\begin{array}{l}\text { Individual sera which, in } 0.1 \text { cc. amounts, } \\
\text { protected against the following doses } \\
\text { of Type II pneumococcus culture, } \\
\text { strain Number } 53\end{array}$} & \multirow{2}{*}{\multicolumn{2}{|c|}{$\underbrace{\text { Number }}_{\text {tested }}$}} & \multicolumn{5}{|c|}{$\begin{array}{l}\text { Individual sera which, in } 0.1 \text { cc. amounts, } \\
\text { protected against the following doses } \\
\text { of Type I pneumococus culture, } \\
\text { strain Number } 1\end{array}$} \\
\hline & & & \multirow{2}{*}{\multicolumn{2}{|c|}{$\frac{-1 \text { M.F.D. }}{2}$}} & \multicolumn{2}{|c|}{100 M.F.D. } & \multicolumn{2}{|c|}{$\begin{array}{l}10,000 \\
\text { M.F.D. }\end{array}$} & & & \multicolumn{2}{|c|}{-1 M.F.D. } & \multicolumn{2}{|c|}{100 M.F.D. } & $\begin{array}{l}10,000 \\
\text { M.F.D. }\end{array}$ \\
\hline Recovered cases & \multicolumn{2}{|c|}{3} & & & \multicolumn{2}{|c|}{$1^{*}$} & & & \multicolumn{2}{|c|}{3} & \multicolumn{2}{|c|}{$3^{*}$} & & & \\
\hline Persistent carriers & F.A. & 5 & F.A. & 2 & $\underset{2}{F . A .}$ & 1 & F.A. & 2 & F.A. & 3 & $\underset{2}{\text { F.A. }}$ & 2 & $\underset{1}{F_{i} A}$. & 1 & F.A. \\
\hline Former carriers & 2 & 9 & $1 \dagger$ & 3 & $1 \ddagger$ & 4 & & 2 & 2 & 2 & & 2 & $1 \ddagger$ & & $1 \dagger$ \\
\hline \multirow{2}{*}{ Total sera of carriers studied } & 5 & 14 & 2 & 5 & 3 & 5 & & 4 & 5 & 5 & 2 & 4 & 2 & 1 & 1 \\
\hline & \multicolumn{2}{|c|}{19} & \multicolumn{2}{|l|}{7} & \multicolumn{2}{|l|}{8} & 4 & & \multicolumn{2}{|c|}{10} & \multicolumn{2}{|l|}{6} & \multicolumn{2}{|l|}{3} & 1 \\
\hline Controls & \multicolumn{2}{|c|}{10} & \multicolumn{2}{|l|}{8} & \multicolumn{2}{|c|}{$2 \S$} & & & \multicolumn{2}{|c|}{10} & \multicolumn{2}{|l|}{8} & \multicolumn{2}{|l|}{2} & \\
\hline
\end{tabular}

F.A. = Received Felton Type I and Type II antigens in 1934.

* Undiluted serum of one person agglutinated the Type II cells and precipitated the supernatant broth culture.

† Skin reaction elicited in this person with Type I polysaccharide, March 31, 1936.

$\ddagger$ Undiluted serum of this person agglutinated Type I cells. Skin reaction elicited with Type I polysaccharide, March 31, 1936.

$\$$ One probably less than 100 M.F.D.

suspensions of the standard Type I and Type II strains were used, and also precipitation tests in which the supernatant fluids from the broth cultures were employed (4). The sera were tested both undiluted and diluted $1: 5$. Reactions were obtained with only two of these sera, and with none of the control sera; the undiluted serum from one of the recovered cases agglutinated the Type II cells and precipitated the supernatant fluid $;^{5}$ the undiluted serum from one former carrier who had received the Felton antigens, agglutinated only the Type I cells.

As a result of the findings obtained during the second survey, a third and final one was made beginning March 31. On this occasion, skin tests were performed according to the method of Tillett and Francis (5), employing pneumococcus typespecific polysaccharides I and II, prepared by Dr. Rachel Brown of this laboratory. Eleven available members of the group of twenty-two whose blood sera were studied during the preceding survey were selected for the tests. None of the men showed any appreciable skin reaction to the Type II polysaccharide, but of four original carriers in

\footnotetext{
- Unfortunately this individual was not available for skin testing in the subsequent investigation.
}

this group who had received the Felton antigens, two developed marked skin reactions within thirty minutes after the injection of the Type I polysaccharide, characterized in one case by wheal and erythema, and in the other, by a large area of erythema alone.

After the completion of the skin testing, the third survey of pneumococcus carriers was undertaken. A certain company in the smaller of these two camps in Orange County was chosen for study since the largest number of persistent carriers had been assigned to this company, and also because of the fact that a severe epidemic of upper respiratory infection had occurred among the men during the early part of March, at which time a number of them required hospitalization in the infirmary. Of the eight original carriers and two former cases assigned to this particular company, among whom four carriers and one case had been found still to harbor Type II pneumococci at the time of the survey in February, all but one were still available for study. Of the four carriers from whose throats Type II pneumococci were isolated in December and again in February, three were found to be carriers of this microörganism in April. None of the other four original carriers could be shown to have reverted to the carrier 
state; one of the two former cases still available for study had apparently ceased to be a carrier since the previous examination.

The throats of seventy other men in the company were cultured. This number represented practically all who were remaining in preparation for evacuating the camp in the middle of April. Eighteen were men transferred from the camp in Madison County who during the survey there in December were not found to be carriers of Type II pneumococci. Most of the others had been transferred in October, 1935, from a camp in Montgomery County where the men were said to have been remarkably free from respiratory infections.

Strains of pneumococcus Type II were isolated from the throats of only two of the seventy men studied. Neither gave a history of contact with patients having pneumonia, and neither had been in the camp in Madison County. Both were housed in a barrack in which none of the proven carriers were lodged. The inference is fairly strong, although by no means conclusive, that these two men developed the carrier state through association with the carriers in the mess hall, in the recreation hall, or in the field. The throats of these two men were recultured two days later, and virulence tests were performed with the two strains of Type II pneumococci isolated from the heart's blood of each inoculated mouse. Furthermore, because of the possibility that the virulence of the strains might have been raised by passage through mice, successful attempts were made in these two instances to isolate Type II pneumococci from blood-agar plates streaked with Avery's broth culture. While in one instance the strain was isolated with considerable difficulty, in the other, no trouble was encountered, for examination of Avery's broth culture by the Neufeld technic indicated that an almost pure culture of Type II pneumococci was present. Strains isolated from the heart's blood of the two mice and directly from the blood-agar plates all proved to be highly virulent; $0.00000001 \mathrm{cc}$. of a 12 -hour broth culture, injected into mice in duplicate, killed them within forty-eight hours; pour plates of this dilution indicated that an average of two microorganisms had been injected.

The relationship between the carrier state and upper respiratory infection in this survey in April was no more evident than in the December survey. Of the three persistent carriers, one was suffering at this time from a head cold; none gave a history of recent respiratory infection. Of the two carriers who were detected for the first time during the April survey, one gave a history of a slight throat irritation some weeks previously, while the other, from whose throat Type II pneumococci were isolated in large numbers, gave a negative history. Of the noncarriers, 14 per cent were suffering from upper respiratory infection; 36 per cent gave a history of a recent cold; and 50 per cent gave no such history.

Although a general survey of the larger of these two camps seemed impractical, four of the men who were found to be carriers both in December and in February were again examined; Type II pneumococci were isolated from the throats of three of them; all four gave a history of very recent respiratory infection.

Quite apart from the main purpose of the investigation, some of the strains of pneumococci isolated from the throats of men who were not found to be carriers of Type II were also studied. Less attention was paid to this feature during the first survey than during the last two when the types of all strains of pneumococci isolated from the heart's blood or peritoneal exudate of each inoculated mouse were identified. Eightyfour, sixteen, and fifty-four strains of pneumococci were isolated and typed during the three successive investigations respectively.

Table III is a recapitulation of the incidence of various types of pneumococci exclusive of strains of pneumococcus Type II isolated from recovered cases. In only six instances was the same type of pneumococcus isolated on more than one occasion from the same individual. Pneumococci Types I, V, VII, and XIV, which frequently incite pneumonia, were not encountered during any of these surveys. On the other hand, Types III and VIII, both important pathogenic agents, were not infrequently observed.

\section{LITERATURE}

Reports in the literature indicate that the occurrence of Type II pneumococci in a normal throat is unusual. Stillman (6) in 1917, examined the saliva of 297 normal individuals, and found pneumococci in 116 instances; pneumococ- 
TABLE III

Types of pneumococci found in throat cultures (excluding strains of Type II from recovered cases)

\begin{tabular}{|c|c|c|c|}
\hline Pneumococcus type & December & February & April \\
\hline 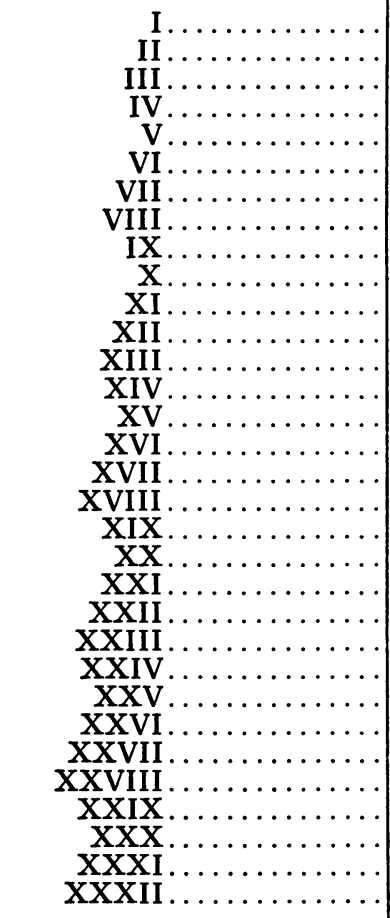 & $\begin{array}{l}4 \\
1 \\
1 \\
2 \\
1 \\
1 \\
1 \\
1 \\
5 \\
1 \\
3 \\
1 \\
2 \\
2 \\
2 \\
1\end{array}$ & $\begin{array}{l}9 \\
1 \\
3\end{array}$ & $\begin{array}{r}8 \\
10 \\
7\end{array}$ \\
\hline $\begin{array}{l}\text { Total numbers of pneumo- } \\
\text { coccus strains, isolated } \\
\text { and typed ................. }\end{array}$ & 84 & 16 & 54 \\
\hline
\end{tabular}

cus Type I was observed in one specimen, and Type II not at all. More recently, Webster and Hughes (7) found pneumococci in about 80 per cent of the 105 healthy adults and children studied. In a total of more than 3,000 cultures, Type II pneumococci were found in but three single cultures from two individuals and Type I was obtained on only one occasion from a single individual.

In order to determine the frequency with which normal individuals in contact with cases of Type I or Type II pneumonia acquire these types of pneumococci, Stillman (6) dispatched a nurse to the home as soon as possible after a patient suffering from pneumonia due to Type I or Type II pneumococcus was admitted to the Rockefeller Hospital. Specimens of saliva were collected from all persons who had come in contact with the patient. Of 107 contacts in a total of twenty- eight households where cases of Type I pneumonia had occurred, 15 per cent were found to harbor Type I pneumococci; of seventy-seven contacts in a total of twenty-four households where cases of Type II pneumonia had occurred, 6 per. cent were found to harbor Type II pneumococci. The average carrying period for Type I was twenty-five days, and for Type II, fortythree days.

Cole (8) states that " in most instances in which pneumococci of Type I or Type II have been found in the mouths of normal persons, these persons have very recently been closely associated with patients suffering from pneumonia due to the same type of pneumococcus as that isolated " and again (9) "One of the most interesting facts which has appeared as a result of the study of the different types of pneumococci is that pneumococci of the so-called fixed types, I and II, which are responsible for such a large proportion. of the cases of pneumonia, are not found in normal mouths, and that within a relatively short time after convalescence they disappear from the mouths of those who have suffered from pneumonia due to one or other of them."

In a study of the epidemiology of pneumonia, Smillie (10) examined strains of pneumococci in the nasopharynx of immediate family contacts, and concluded from his series of 582 contacts and 493 controls that Types I and II were much more prevalent in the nasopharynges of contacts than in the population at large. This finding did not prove to be true with regard to many of the types of pneumococci which were formerly included in Group IV.

The epidemic nature of lobar pneumonia and the probable rôle of the carrier has been discussed by Gundel and Wallbruch $(11,12)$ in a survey of a small village of three hundred inhabitants near Berlin, where an epidemic of Type I pneumonia had occurred during February, 1935. This outbreak followed two weeks after an epidemic of "grippe"; the first case developed in the village school, where the disease spread rapidly to other pupils and then among their respective families. These authors have convincingly traced the spread of the disease from one individual to the next, through the medium of cases and carriers, of which there were a large number 
among the examined contacts. On the basis of these findings and of the few similar reports in the literature, these authors emphasize that lobar pneumonia should be classed as a true contagious disease, which may, under certain circumstances, become epidemic.

With regard to the serological studies in connection with the carrier condition, Tilghman and Finland (13) have reported suggestive findings in tests performed with the blood of individuals in contact with cases of pneumonia, comparable to the results described in this paper. They have shown that specific antibodies may develop-in individuals who are carriers of the diseaseproducing pneumococcus but who do not become ill with the infection.

\section{SUMMARY AND CONCLUSIONS}

An epidemic of respiratory infection in a veterans' camp of the Civilian Conservation Corps, located in Madison County in New York State, was followed by an outbreak of lobar pneumonia, in which the mortality rate was low; only one of nine cases died (Type II pneumococci were isolated from the blood of two of the patients who recovered).

After the outbreak of pneumonia, an investigation was undertaken to determine the incidence and distribution of carriers of Type II pneumococci within the camp. Nineteen per cent of the enrolled men living in the barracks were found to be carriers of this microörganism.

The last case of Type II pneumonia at this camp developed three weeks after the next preceding one. This lapse of time, together with the failure to demonstrate the presence of Type II pneumococci in the throat only six days before the onset of pneumonia, lends strong support to the belief that the infection was acquired in this instance not from one of the cases but rather through contact with a carrier, not unlikely the carrier in the adjoining bed.

Ten weeks after the conclusion of the first investigation, a second was undertaken in camps in Orange County to which most of the campers had been transferred. The carrier state was found to persist in a number of the original carriers. Tests with the isolated strains of Type II pneumococci revealed a high degree of virulence for mice. Serological tests with the blood of carriers suggested that in certain instances there may have been a significant correlation between the protective activity of individual blood serum against the Type II pneumococcus and the presence or recent existence of Type II pneumococci in the throat. Protective activity against the Type I pneumococcus was, on the whole, more evident in the group of sera from those who had received the Felton Type I and Type II antigen in 1934, than in the sera from those who had not; on the other hand, such evidence was lacking with regard to protection against Type II. The small number of sera examined prevents the drawing of definite conclusions. Skin tests, performed five weeks later, with polysaccharides Types I and II yielded reactions to Type I in only two of the group treated by Felton.

A final survey was undertaken fifteen weeks after the conclusion of the first survey. A number of the original carriers were found still to harbor Type II pneumococci in the throat, and two men who, as far as is known had come in contact with no case of Type II pneumonia, were shown in this survey to be carriers of virulent Type II pneumococci, thus suggesting that the carrier state in these instances had developed through contact with other carriers. In view of the persistence and the apparent spread of the Type II carrier condition, it is noteworthy that although an epidemic of respiratory infection seemed to have prepared the way for an outbreak of Type II pneumonia in the Madison County camp, a similar epidemic in the camp in Orange County was followed by no cases of Type II pneumonia.

These investigations lend support to the accumulating evidence that the carrier is an important factor in the transmission of pneumococcus infection. On the other hand, the question of why certain individuals develop pneumonia on exposure to virulent pneumococci while others become carriers, can not be adequately explained on the basis of the studies of host resistance.

We are indebted to Dr. William A. Wall, who provided the facilities of his laboratory during the first study, and to the medical officers of the camps: Lieutenant Colonel Blank, Lieutenants Stein, Troupin, and Freeman. 


\section{BIBLIOGRAPHY}

1. Wadsworth, A. B., Standard Methods of the Division of Laboratories and Research of the New York State Department of Health. Williams and Wilkins Co., Baltimore, 1927, p. 110.

2. Kirkbride, M. B., Hendry, J. L., and Murdick, P. P., The concentration and standardization of Type I antipneumococcus serum. Am. J. Hyg., 1936, 23, 187.

3. Felton, L. D., Possibility of prophylaxis against pneumococcus infections. Second Internat. Congress for Microbiology, Abstracts of communications. London, 1936, p. 293.

4. Wadsworth, A. B., Reference (1), p. 427.

5. Tillett, W. S., and Francis, T., Jr., Cutaneous reactions to the polysaccharides and proteins of pneumococcus in lobar pneumonia. J. Exper. Med., 1929, 50, 687.

6. Stillman, E. G., Further studies on the epidemiology of lobar pneumonia. J. Exper. Med., 1917, 26, 513.

7. Webster, L. T., and Hughes, T. P., The epidemiology of pneumococcus infection. The incidence and spread of pneumococci in the nasal passages and throats of healthy persons. J. Exper. Med., 1931, 53, 535.

8. Cole, R., Acute Lobar Pneumonia. Nelson Loose Leaf Living Med., Thomas Nelson and Sons, New York, 1920, 1, p. 203.

9. Cole, R., Acute Lobar Pneumonia. Nelson Loose Leaf Living Med., Thomas Nelson and Sons, New York, 1929, p. 104.

10. Smillie, W. G., The epidemiology of lobar pneumonia. A study of the prevalence of specific strains of pneumococci in the nasopharynx of immediate family contacts. J. A. M. A., 1933, 101, 1281.

11. Gundel, M., and Wallbruch, E., Die lobäre Pneumonie als epidemisch auftretende Krankheit. Deutsche med. Wchnschr., 1935, 61, 539.

12. Wallbruch, E., Die lobäre Pneumonie als epidemisch auftretende Erkrankung. Ztschr. f. Hyg. u. Infektionskr., 1935, 117, 92.

13. Tilghman, R. C., and Finland, M., Multiple cases of pneumococcic infections in families with the demonstration of the development of antibodies in contact carriers. J. Clin. Invest. (Proc.), 1936, 15, 471. 